\title{
Editorials
}

Pneumonia is a common cause of morbidity and mortality. In the United Kingdom community acquired pneumonia has an estimated incidence of one to three cases per 1000 adults per year and is responsible for over 60000 deaths each year. ${ }^{1}$ Adequate management requires the selection of appropriate antibiotics and studies have shown that the use of inappropriate agents is associated with increased mortality. ${ }^{23}$ The selection of antibiotics is guided by the identification of the causative pathogen, which in the case of community acquired pneumonia is achieved in only 30 $80 \%$ of patients, depending on the population studied and the investigative techniques used. There are various reasons to account for the failure to identify pathogens: specimens may not be obtained from patients, organisms may not be cultured (perhaps because of the use of antibiotics before collection of specimens), or specific tests may not be routinely performed (such as serological testing for Chlamydia pneumoniae). Even when pathogens are identified results may not be available for 48 hours or longer after hospital admission. Because of the shortfalls in current diagnostic methods alternative techniques that might increase diagnostic yield, provide rapid results, and not be affected by prior antibiotics have attracted interest. Some of this interest has centred on the detection of antigens shed by pathogens.

\section{Streptococcus pneumoniae}

Streptococcus pneumoniae is the most common causative pathogen for community acquired pneumonia. With detailed investigations it can be found in up to $76 \%$ of patients admitted to hospital ${ }^{4}$ but in routine clinical practice it is identified less frequently. Blood culture remains the diagnostic gold standard by which other techniques are measured but few patients with pneumonia develop bacteraemia. Blood cultures therefore may be positive in only $25-30 \%$ of cases. ${ }^{2}$ Sputum microscopy and culture for $S$ pneumoniae are bedevilled with the problem of specificity. As the oropharynx may be colonised by $S$ pneumoniae in $35 \%$ of children, $20 \%$ of healthy adults, and $40 \%$ of adults with chronic bronchitis ${ }^{56}$ contamination of expectorated sputum makes it difficult to decide whether cultured $S$ pneumoniae comes from the lower or the upper respiratory tract. Counts of diplococci and leucocytes seen by sputum microscopy may help to make this distinction and may raise specificity to $85 \%$ and sensitivity to $62 \% .^{7}$ An alternative diagnostic approach is to look for the development of antibodies but this delays results. ${ }^{8}$

$S$ pneumoniae elaborates various toxins and type or species specific surface markers (table 1). These toxins and surface markers are immunogenic and are detected by immunoassays. There has therefore been interest in using immunoassays for these antigens to improve the diagnostic rate for pneumococcal infection. Apart from recent work on antibody responses to the toxin pneumolysin' most work has been on two antigens, both of which are polysaccharides. Pneumococcal C polysaccharide is a teichoic acid, a major constituent of the cell wall. It is species but not type specific, and is the ligand for $\mathrm{C}$ reactive protein. Pneumococcal capsular polysaccharide antigens form the outer surface of the organism and are type specific, with 83 different serotypes. Twenty three common pneumococcal capsular polysaccharide antigens comprise the current 23 valent pneumococcal vaccine.

\section{Community acquired pneumonia}

Several studies have now shown that pneumococcal C polysaccharide and pneumococcal capsular polysaccharide antigen can be detected during active pneumococcal infection in various body fluids. They include studies on community acquired pneumonia from diverse geographical locations in West Africa, ${ }^{1011}$ Europe, ${ }^{2412-16}$ and the United States. ${ }^{17}$ They have differed in the technique used for antigen detection, the nature of the antigen sought, the age group of patients, and the selection of patients. Most of these studies, however, have used countercurrent immunoelectrophoresis to detect pneumococcal capsular polysaccharide antigen with Omniserum (Statens Serum Institut, Copenhagen) in bacteraemic and non-bacteraemic adults. Three important conclusions may be drawn.

Firstly, pneumococcal antigens are distributed widely in body fluids and are found in sputum, pleural fluid, serum, and urine during acute pneumococcal pneumonia. The frequency of antigen detection in sputum, serum, and urine in six studies is shown in table 2 . Pneumococcal antigen is most often found in sputum, followed by urine. The frequency with which pneumococcal antigen is present in serum varies considerably between studies and may reflect the handling of specimens and the sensitivity of the assays.

Secondly, pneumococcal antigens may persist in serum and urine long after the onset of pneumonia. For example pneumococcal capsular polysaccharide antigen, as detected by latex agglutination, has been found in the urine of two patients 25 weeks after admission to hospital. ${ }^{14}$. This

Table 1 Surface antigens and toxins of Streptococcus pneumoniae

Capsule

Pneumococcal capsular polysaccharide antigens

Cell wall

Pneumococcal C polysaccharide

$M$ protein antigen

$R$ protein antigen

Toxins

Pneumolysin

Purpura producing principle

Neuraminidase

Autolysins

Table 2 Frequency of pneumococcal antigen detection in sputum, serum, and urine in six studies of community acquired pneumonia

\begin{tabular}{llcl}
\hline & \multicolumn{3}{l}{ Percentage of samples } \\
\cline { 2 - 4 } Reference & Sputum & Serum & Urine \\
\hline British Thoracic Society ${ }^{2}$ & 86 & 9 & 45 \\
Macfarlane et al $^{4}$ & 72 & 11 & 64 \\
Tugwell et al ${ }^{10}$ & 79 & 29 & 54 \\
Ortqvist et al $^{12}$ & 68 & - & - \\
Boersma et al ${ }^{16}$ & 94 & 41 & - \\
Coonrod et al ${ }^{17}$ & - & 41 & - \\
\hline
\end{tabular}


persistence has been explained by the steady release of unmetabolised antigen from tissue depots and phagocytic cells. ${ }^{18}$ Clearance of antigen is aided by specific antibody but clearance of pneumococcal capsular polysaccharide antigen is very dependent on capsular type. ${ }^{19}$

Thirdly, antigenaemic patients are clinically more unwell than non-antigenaemic patients. ${ }^{10}{ }^{17}$ Antigenaemic patients are more likely to develop complications and have persistent pyrexia, and they tend to require longer in hospital. This clinical correlation may be due to the association between antigenaemia and bacteraemia, though serum antigen is not directly derived from circulating bacteria ${ }^{17}$; the number of circulating bacteria is too small to account for the concentration of serum antigen, which must therefore come directly from pneumonic lung.

\section{Diagnosis}

The detection of pneumococcal antigens is unaffected by the prior use of antibiotics and assays are rapid, providing results on the same day. This is encouraging for the use of pneumococcal antigens in diagnosing pneumococcal infections, but does antigen detection provide specific and sensitive diagnostic information?

The diagnostic specificity for pneumococcal infection of pneumococcal antigens in serum or urine is undoubted. Not all patients with pneumonia become antigenaemic, however, and so there is a limit to the sensitivity. The use of urine is more sensitive than serum, presumably because urine samples are concentrated, the use of an absorbent gel before the assay making it easier to detect very small quantities. The relative persistence of antigen in serum and urine is useful because specimens can be collected late as well as early in the course of infection, though with a declining yield of positive results.

Potentially, sputum assay could contribute considerably to diagnostic yield given the high frequency of pneumococcal antigens in sputum. There is uncertainty, however, about the diagnostic specificity of pneumococcal antigens found in sputum. As with sputum culture, expectorated samples could conceivably be contaminated by antigen derived from $S$ pneumoniae colonising the oropharynx. It is impossible therefore to be certain whether pneumococcal antigen in sputum is derived from the upper or the lower respiratory tract.

This problem has been addressed by a recent study (submitted for publication). This has shown that oropharyngeal organisms, in the absence of active lower respiratory tract infection, produce antigens in saliva that give positive reactions with Omniserum in about $20 \%$ of healthy volunteers, inpatients, and outpatients. Most of these reactions are non-specific and can be removed by prior absorption of Omniserum with viridans streptococci, but in a few cases the organisms are typable and presumably the reactions are due to oropharyngeal $S$ pneumoniae. Oropharyngeal contamination of expectorated sputum is therefore a potential problem. One way of dealing with this problem is to wash sputum samples to reduce any contamination. ${ }^{16}$ Two important clues, however, indicate that during pneumonia pneumococcal capsular polysaccharide antigen in expectorated, unwashed sputum comes principally from the lower respiratory tract. Firstly, the serotype of sputum pneumococcal capsular polysaccharide antigen is the same as the serotype of blood culture isolates. ${ }^{10}$ Secondly, the concentration of pneumococcal capsular polysaccharide antigen in sputum considerably exceeds that in concomitant saliva, making it unlikely that the antigen in sputum is derived from contamination. More work needs to be done to calculate the diagnostic specificity and sensitivity of sputum assay. Unfortunately the latter cannot be used in all patients with pneumonia because about $30 \%$ do not expectorate sputum. ${ }^{2}$

Pneumococcal C polysaccharide ${ }^{20}$ and pneumococcal capsular polysaccharide antigen (work submitted for publication) are also detectable in saliva during acute pneumonia. Saliva is a readily available specimen and could prove useful for diagnosis if sputum is unavailable. A distinction must again be made, however, between local oropharyngeal production of antigen and antigen derived from carriage up the mucociliary escalator from the lower respiratory tract. As with sputum pneumococcal capsular polysaccharide antigen, there is a concordance of serotypes between salivary pneumococcal capsular polysaccharide antigen and blood culture isolates. This suggests that salivary pneumococcal capsular polysaccharide antigen may indicate a pathogen but further work is required on its specificity.

Measurement of the antibody response to pneumolysin gives delayed results and is best reserved for epidemiological studies. ${ }^{911}$ It has proved to be specific, with a sensitivity of $80-90 \%$.

\section{Immunoassays}

A range of immunoassays is available but there are three basic techniques: countercurrent immunoelectrophoresis, agglutination, and enzyme linked immunosorbent assay (ELISA). Comparative studies on these techniques have been performed ${ }^{121421-23}$ and ELISA has proved the most sensitive and countercurrent immunoelectrophoresis the least sensitive. Each technique has its own limitations. Countercurrent immunoelectrophoresis does not readily detect positively charged pneumococcal capsular polysaccharide antigens, such as types 7 and 14. Latex agglutination is relatively poor at detecting antigen in urine for various reasons. ${ }^{23} 24$ ELISA is the most expensive and time consuming technique and is best reserved for batch testing. All of these techniques are also limited by the quality of antiserum used; cross reactions with non-pneumococcal organisms have been reported for antisera against pneumococcal $C$ polysaccharide ${ }^{25}$ and pneumococcal capsular polysaccharide antigen. ${ }^{26}$ When these immunoassays are applied to sputum diagnosis the increased sensitivity may result in false positives and decreased specificity. A practical approach therefore would be to use agglutination or countercurrent immunoelectrophoresis for clinical specimens and reserve ELISA for the batch testing of research samples.

\section{Conclusions}

Pneumococcal antigens are readily detected in various body fluids during acute and convalescent pneumococcal pneumonia. Assay for these antigens holds considerable promise in improving the diagnostic rate in pneumococcal infections. The diagnostic specificity of serum, pleural fluid, and urine assay is very high and sensitivity is greater than for cultural methods. For sputum assay the sensitivity is $68-94 \%$ and accumulating evidence suggests that the specificity for pneumococcal pneumonia is good. More work is required to define the usefulness of saliva assay. At the moment antigen detection is helpful in aetiological research studies and in the clinical setting of an unwell patient whose initial cultures are unhelpful. The widespread, routine use of antigen detection must await further research identifying the best antigen to assay, the optimal assay technique, and which specimens to collect and when. Antigen detection should reduce the percentage of patients with pneumonia for whom a causative pathogen is not 
identified and thereby reduce the dependence on empirical antimicrobial treatment.

\section{P VENKATESAN \\ Department of Microbiology J T MACFARLANE \\ Department of Respiratory Medicine}

City Hospital

Nottingham NG5 1PB

Reprint requests to: Dr J T Macfarlane.

1 Macfarlane JT. Community-acquired pneumonia. Br J Dis Chest 1987; 81:116-27.

2 Research Committee of British Thoracic Society and Public Health Laboratory Service. Community-acquired pneumonia in adults in British hospitals 1982-1983: a survey of aetiology, mortality, prognostic factors and outcome. $Q J$ Med 1987;239:195-220.

3 Marrie TJ, Durant H, Yates L. Community-acquired pneumonia requiring hospitalization: 5-year prospective study. Rev Infect Dis 1989;11:586-99.

4 Macfarlane JT, Finch RG, Ward MJ, Macrae AD. Hospital study of adult community-acquired pneumonia. Lancet 1982;ii:255-8.

5 Hendley JO, Sande MA, Stewart PM, Gwaltney JM. Spread of Streptococcus pneumoniae in families. I. Carriage rates and distribution of types. $J$ Infect Dis 1975;132:55-61.

6 Haas H, Morris JF, Samson S, Kilbourn JP, Kim PJ. Bacterial flora of the respiratory tract in chronic bronchitis: comparison of transtracheal, fiberbronchoscopic, and oropharyngeal sampling methods. Am Rev Respir Dis 1977;116:41-7.

7 Rein MF, Gwaltney JM, O'Brien WM, Jennings RH, Mandell GL. Accuracy of the Gram's stain in identifying pneumococci in sputum. JAMA 1978;239:2671-3.

8 Löwenberg A, Snidjer JAM, Weele LT, Sluiter HJ. Pneumococcal antibody levels in patients with acute lung infiltrates. Infection 1987;15:192-9.

9 Jalonen E, Paton JC, Koskela M, Kerttula Y, Leinonen M. Measurement of antibody response to pneumolysin-a promising method for the aetiological diagnosis of pneumococcal pneumonia. J Infect 1989;19: 127-34.

10 Tugwell P, Greenwood BM. Pneumococcal antigen in lobar pneumonia. J Clin Pathol 1975;28:118-23.

11 Forgie IM, O'Neill KP, Lloyd-Evans $N$, Leinonen $M$, Campbell $H$, Whittle HC, et al. Etiology of acute respiratory tract infections in Gambian children: II. Acute lower respiratory tract infections in children ages one to nine years presenting to the hospital. Pediatr Infect Dis J 1991;10:42-7.

12 Ortqvist A, Jönsson I, Kalin M, Krook A. Comparison of three methods for detection of pneumococcal antigen in sputum of patients with communityacquired pneumonia. Eur J Clin Microbiol Infect Dis 1989;8:956-61.

13 Krook A. Holmberg H. Pneumococcal antigens in sputa: ELISA for the detection of pneumococcal C-polysaccharide in sputa from pneumonia patients. Diagn Microbiol Infect Dis 1986;7:73-5.

14 Van der Auwera P, Andre A, Bulliard G, Legrand JC, Gordts B, Van Landuyt $\mathrm{H}$, et al. Comparison of latex agglutination and counterimmunoelectrophoresis in the diagnosis of acute Streptococcus pneumoniae infections. Eur J Clin Microbiol 1983;2:534-40.

15 Woodhead MA, Macfarlane JT, McCracken JS, Rose DH, Finch RG. Prospective study of the aetiology and outcome of pneumonia in the community. Lancet 1987;i:671-4.

16 Boersma WG, Löwenberg A, Holloway Y, Kutschrütter H, Snidjer JAM, Koëter GH. Pneumococcal capsular antigen detection and pneumococcal serology in patients with community acquired pneumonia. Thorax 1991; 46:902-6.

17 Coonrod JD, Drennan DP. Pneumococcal pneumonia: capsular antigenaemia and antibody responses. Ann Intern Med 1976;84:254-60.

18 Howard JG, Christie GH, Jacob MJ, Elson J. Studies on immunological paralysis. III. Recirculation and antibody-neutralising activity of ${ }^{14} \mathrm{C}$-labelled type III pneumococcal polysaccharide in paralysed mice. Clin Exp Immunol 1970;7:583-96.

19 Schaffner A, Michel-Harder C, Yeginsoy S. Detection of capsular polysaccharide in serum for the diagnosis of pneumococcal pneumonia: clinical and experimental evaluation. J Infect Dis 1991;163:1094-102

20 Krook A, Fredlund H, Holmberg $H$. Diagnosis of pneumococcal pneumonia by detection of antigen in saliva. Eur J Clin Microbiol 1986;5:639-42.

21 Edwards EA, Coonrod JD. Coagglutination and counterimmunoelectrophoresis for detection of pneumococcal antigens in the sputum of pneumonia patients. J Clin Microbiol 1980;11:488-91.

22 Lenthe-Eboa S, Brighouse G, Auckenthaler R, Lew D, Zwahlen A, Lambert $\mathrm{PH}$, et al. Comparison of immunological methods for diagnosis of pneumococcal pneumonia in biological fluids. Eur J Clin Microbiol 1987; 6:28-34.

23 Woodhead MA, Macfarlane JT, Finch RG, McCracken JS. A comparison of countercurrent immunoelectrophoresis and latex agglutination for the detection of pneumococcal antigen in a community based pneumonia study. Serodiagn Immunother Infect Dis 1990;4:159-65.

24 Lebrun L, Guibert M, Pillot J. Failure of tests to detect pneumococcal antigens in urine. Eur J Clin Microbiol Infect Dis 1988;7:83-4.

25 Sjögren AM, Holmberg H, Krook A. Etiologic diagnosis of pneumonia by antigen detection: crossreactions between pneumococcal C-polysaccharide and oral microorganisms. Diagn Microbiol Infect Dis 1987;6: 239-48.

26 Holmberg $H$, Danielsson D, Hardie J, Krook A, Whiley $R$ Cross-reactions between $\alpha$-streptococci and omniserum, a polyvalent pneumococcal serum, demonstrated by direct immunofluorescence, immunoelectroosmophoresis, and latex agglutination. J Clin Microbiol 1985;21:745-8. 